\title{
Boundary scattering and weak localization of electrons in a magnetic field
}

\author{
C. W. J. Beenakker and H. van Houten \\ Philips Research Laboratories, $5600 \mathrm{JA}$ Eindhoven, The Netherlands
}

(Received 2 October 1987)

\begin{abstract}
The influence of boundary scattering on one- and two-dimensional weak localization is studied both analytically and by numerical simulation. Diffuse and specular boundary scattering are considered for two geometries, a metal film in a parallel magnetic field and a laterally restricted twodimensional electron gas in a perpendicular field. The results, which extend the Al'tshuler-Aronov and Dugaev-Khmel'nitskii theories, are relevant to recent magnetoresistance experiments on highmobility channels in $\mathrm{GaAs}-\mathrm{Al}_{x} \mathrm{Ga}_{1-x}$ As heterostructures.
\end{abstract}

\section{INTRODUCTION}

Weak localization of electrons is a quantum interference effect which enhances the probability of return by classical diffusion and thus reduces the conductivity. ${ }^{1}$ This phenomenon is due to the constructive interference of a closed electron path and its time reverse. A magnetic field destroys the phase coherence of these two paths, resulting in an increased conductivity. For a thin film in a perpendicular field $B$, phase coherence is lost after a time $\tau_{B} \sim \hbar / e B D$ ( $D$ is the diffusion coefficient). On this time scale a flux $B D \tau_{B}$ of order $\hbar / e$ is enclosed, corresponding to a phase difference of order 1 between a closed path and its time reverse. In a parallel field the problem is complicated by boundary scattering. If the (bulk) elastic mean-free path $l_{e}$ is much smaller than the film thickness $W$, the boundaries simply restrict the diffusive motion in one direction so that the enclosed flux is $B W\left(D \tau_{B}\right)^{1 / 2}$, leading to the estimate $\tau_{B} \sim(\hbar /$ $e B W)^{2} / D$. This is the dirty-metal regime treated by the Al'tshuler-Aronov (AA) theory. ${ }^{2}$ The pure-metal regime $l_{e} \gg W$ was studied by Dugaev and Khmel'nitskii ${ }^{3}$ (DK) for the case of diffuse surface scattering in the limits of small and large magnetic fields. A characteristic feature of this regime, in which electrons move ballistically from one surface to the other, is the flux cancelation ${ }^{4}$ shown in Fig. 1. Since closed trajectories involving only wall col-

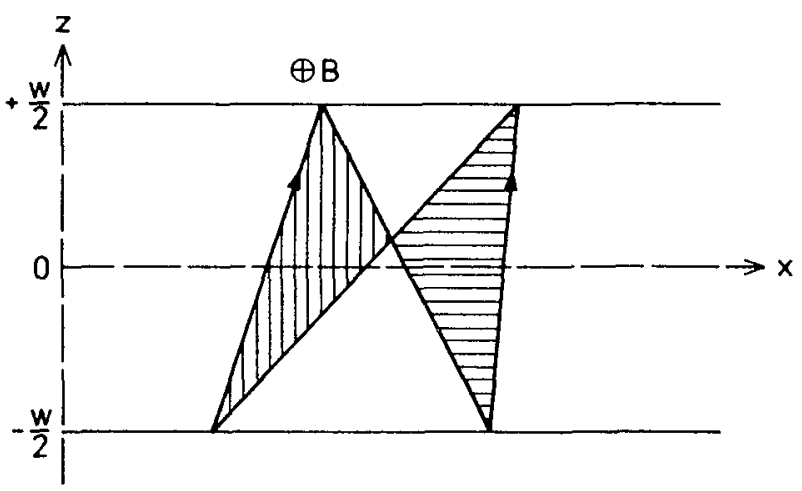

FIG. 1. Closed trajectory of one electron in a thin film or narrow channel, illustrating the characteristic flux cancelation. (The shaded areas are exactly equal and of opposite orientation.) lisions enclose zero flux, impurity collisions are necessary for phase relaxation. In the AA theory, on the contrary, impurity scattering hinders phase relaxation by reducing $D$.

It is the purpose of the present paper to extend the AA and DK theories in the three following ways. (1) Beyond the asymptotic regimes of small and large mean-free paths and magnetic fields. The crossover between the regimes described by the AA and DK theories can then be investigated, which is relevant for experiments since these regimes are usually not well separated. (2) To include specular as well as diffuse boundary scattering. This is of importance in the pure-metal regime, where the phase relaxation rate depends on the type of boundary scattering. (3) To include a narrow channel as well as a thin film geometry (one- and two-dimensional weak localization). Our theoretical work was motivated by a recent experiment ${ }^{5}$ in the pure-metal regime. It was shown in Ref. 5 that the magnetoresistance of a narrow GaAs$\mathrm{Al}_{x} \mathrm{Ga}_{1-x} \mathrm{As}$ heterostructure in a perpendicular field could be explained by the results for a channel with specular boundary scattering obtained in the present article. Two methods are used in our analysis. Analytic formulas valid in the asymptotic regimes are derived by means of the simple and elegant "method of trajectories" devised by De Gennes and Tinkham ${ }^{4}$ for the related problem of the parallel critical field of thin superconducting films. (Dugaev and $\mathrm{Khmel}$ 'nitskii ${ }^{3}$ use an alternative method based on a Boltzmann-type kinetic equation.) Numerical results for the intermediate regimes are obtained by a computer simulation of the phase relaxation of an electron in a magnetic field. Since the weak localization effect involves only a very small fraction of the trajectories generated (those which return to the point of departure), this approach would seem prohibitively time consuming. Fortunately, it turns out that the problem can be transformed into one which involves all trajectories, and then simulation is a quick and easy way to calculate $\tau_{B}$. We shall refer to this transformation, which requires choosing a special vector potential, as the "gauge trick."

The outline of the paper is as follows. In Sec. II we formulate the basic equations for the weak localization effect in one and two dimensions. In Sec. III the gauge 
trick mentioned above is introduced. Analytical and numerical results for the phase relaxation time in a magnetic field are given in Secs. IV and V, respectively. We conclude in Sec. VI with a discussion of our results in relation to experiment.

\section{FORMULATION OF THE PROBLEM}

The weak localization effect causes a correction $\Delta \sigma$ to the classical conductivity $\sigma_{0}$ of the form ${ }^{2,3,6}$

$$
\begin{aligned}
\Delta \sigma=-\frac{\sigma_{0}}{\pi \hbar N(0)} \int_{0}^{\infty} d t C(t) e^{-t / \tau_{\phi}} & \\
& \times\left\langle e^{i \phi(t)} \mid \mathbf{R}(t)=\mathbf{R}(0)\right\rangle,
\end{aligned}
$$

where $N(0)$ is the density of states per spin direction at the Fermi level. (Additional degeneracies, such as the valley degeneracy in $\mathrm{Si}$, are ignored.) The integrand is the product of three terms: (1) The classical probability density $C(t)$ of return to the point of departure after a time $t ;(2)$ a damping factor with relaxation time $\tau_{\phi}$ to account for processes which destroy the phase coherence between pairs of time-reversed paths in the absence of a magnetic field, such as inelastic scattering; and (3) a factor containing the phase difference $\phi$ between timereversed paths acquired in a magnetic field. This last term is the conditional average over all closed classical trajectories at time $t$ of $e^{i \phi(t)}$, the phase difference $\phi$ being given by the line integral of the vector potential along the path

$$
\phi(t)=\frac{2 e}{\hbar} \int_{\mathbf{R}(0)}^{\mathbf{R}(t)} \mathbf{A} \cdot d l .
$$

Since in Eq. (2.1) initial and final positions $\mathbf{R}(t)$ and $\mathbf{R}(0)$ coincide, the phase difference depends only on the enclosed flux and is gauge invariant. One can also verify that the average phase factor is real, since the contributions from two identical closed loops traversed in opposite directions are each other's complex conjugates, so that the imaginary part cancels.

The classical trajectories referred to above are realizations of what Chakravarty and Schmid ${ }^{6}$ have termed "Boltzmannian motion" (because of the equivalence with the Boltzmann equation in the relaxation-time approximation): Motion between impurity collisions is ballistic; impurity scattering is elastic and isotropic, and occurs with probability $d t / \tau_{e}$ in a small time increment $d t$. As discussed in Ref. 6, this semiclassical description of weak localization is entirely equivalent to the usual diagrammatic perturbation expansion of the Green's function, in which it is assumed that the Fermi wavelength is much smaller than the mean-free path. In the present case we require in addition, for the semiclassical description to apply, that the Fermi wavelength is much smaller than the sample width.

To calculate $\Delta \sigma$, note that in 1 and 2 dimensions the long-time behavior of the integrand in Eq. (2.1) is most important. In the long-time regime the classical motion of the electrons is diffusive, and the probability density of return is given by $C(t)=(4 \pi D t)^{-d / 2} W^{d-3}$. Here $d=1,2$ is the effective dimensionality of the sample and $W$ its transverse size. The diffusion coefficient $D$ is related to the classical conductivity by the Einstein relation $\sigma_{0}=2 e^{2} N(0) D$. The diffusive approximation for $C(t)$ holds for times much longer than the mean time $\tau_{e}$ between elastic collisions, while for shorter times $C(t)$ goes to zero. One can account for this cutoff at $t \sim \tau_{e}$ in an $a d$ hoc way by inserting the factor $1-\exp \left(-t / \tau_{e}\right)$, thereby excluding those electrons which have not yet been scattered elastically. Substitution into Eq. (2.1) gives the formula

$$
\begin{gathered}
\Delta \sigma_{d}=-\frac{2 e^{2}}{\pi \hbar} D \int_{0}^{\infty} d t(4 \pi D t)^{-d / 2}\left(1-e^{-t / \tau_{c}}\right) e^{-t / \tau_{\phi}} \\
\times\left\langle e^{\prime \phi(t)} \mid \mathbf{R}(t)=\mathbf{R}(0)\right\rangle .
\end{gathered}
$$

Here $\sigma_{d} \equiv \sigma W^{3-d}$ is the conductance of a square for $d=2$, or a unit length for $d=1$. In the absence of a magnetic field $[\phi(t) \equiv 0]$ one finds

$$
\begin{aligned}
& \left.\Delta \sigma_{2}=-\frac{e^{2}}{2 \pi^{2} \hbar} \ln \mid \frac{\tau_{\phi}}{\tau_{e}}+1\right), \\
& \left.\Delta \sigma_{1}=-\frac{e^{2}}{\pi \hbar} \sqrt{D} \mid \tau_{\phi}^{1 / 2}-\left(\frac{1}{\tau_{\phi}}+\frac{1}{\tau_{e}}\right)^{-1 / 2}\right] .
\end{aligned}
$$

The above expressions follow from a particular choice ${ }^{7}$ for the short-time cutoff, but are independent of this choice for $\tau_{\phi} \gg \tau_{e}$. In the geometries given below the average phase factor in a magnetic field $B$ decays exponentially as $\exp \left(-t / \tau_{B}\right)$, with a relaxation time $\tau_{B}$ [see the argument leading to Eq. (3.6) in Sec. III]. Upon substitution into Eq. (2.3) it follows that $\Delta \sigma_{d}$ for nonzero $B$ is given by Eq. (2.4) with $\tau_{\phi}$ replaced by $\left(1 / \tau_{\phi}\right.$ $\left.+1 / \tau_{B}\right)^{-1}$.

In Secs. IV and $V$ we will calculate $\tau_{B}$ for two geometries: (1) A metal film of thickness $W$ containing a three-dimensional electron gas in a parallel field and (2) a channel of width $W$ containing a two-dimensional electron gas in a perpendicular field. The effective dimensionalities are, respectively, $d=2$ and 1 . For each geometry we consider the two extreme cases of specular and diffuse boundary scattering. We assume low temperature and weak magnetic fields so that on the phase relaxation time scale the electrons have many collisions with impurities and boundaries, that is to say $\tau_{\phi}, \tau_{B} \gg \tau_{e}$ and $\tau_{\phi}, \tau_{B} \gg W^{2} / D$. (We will see later that the resulting restriction on the magnetic field is $B \ll \hbar / e W^{2}$.) Since our approach is semiclassical, we also require that the Fermi wavelength $\hbar / m v_{F}$ is much smaller than $W$. In the above magnetic field range the cyclotron radius $m v_{F} / e B$ is then much larger than $W$, so that the curvature of trajectories can be neglected. Before proceeding with the actual calculations we first introduce a technical trick, which we will need.

\section{THE GAUGE TRICK}

The weak localization correction to the conductivity [Eq. (2.3)] contains the phase factor averaged over those trajectories which in a given time $t$ return to their point of departure. The restriction to closed paths very much complicates the (analytical or numerical) calculations. 
We therefore ask the following question: Can one omit this restriction and average over all paths of duration $t$ ? The answer is yes, but only in a special gauge. The appropriate choice for the vector potential is $A=(B z, 0,0)$, with the boundaries of the system at $z= \pm W / 2$ and the magnetic field pointing in the $y$ direction (Fig. 1). That this gauge does the trick can be seen as follows.

We define the function

$$
K\left(\mathbf{r}, \mathbf{r}^{\prime} ; t\right) \equiv\left\langle e^{i \phi(t)} \mid \mathbf{R}(0)=\mathbf{r}, \mathbf{R}(t)=\mathbf{r}^{\prime}\right\rangle,
$$

which is the average of the phase factor over all classical trajectories going from $\mathbf{r}$ to $\mathbf{r}^{\prime}$ in a time $t$. The conductivity (2.3) depends on the value $K(\mathbf{r}, \mathbf{r} ; t)$ for coincident arguments, which is gauge invariant - although $K$ itself is not. We can use the freedom we have in choosing the vector potential to make the quantity $K$ independent of initial and final coordinates, in the long-time regime of diffusive motion. We shall show that this is achieved in the gauge defined above.

In the regime $\tau_{B} \gg \tau_{e}, W^{2} / D$ considered, the dependence of the function $K$ on the $z$ components of $\mathbf{r}$ and $\mathbf{r}^{\prime}$ can be neglected, since the time scale on which $K$ varies is much longer than the time during which a variation in $z$ affects the trajectories. It therefore suffices to consider the average over $z$ of $K$,

$\bar{K}\left(\mathbf{r}_{\|}, \mathbf{r}_{\|}^{\prime} ; t\right) \equiv \int_{-W / 2}^{+W / 2} \frac{d z}{W} \int_{-W / 2}^{+W / 2} \frac{d z^{\prime}}{W} K\left(\mathbf{r}, \mathbf{r}^{\prime} ; t\right)$,

where $\mathbf{r}_{\|} \equiv(x, y)$. For any choice of the vector potential, $\bar{K}$ satisfies the identity

$$
\bar{K}\left(\mathbf{r}_{\|}, \mathbf{r}_{\|}^{\prime} ; t\right)=\bar{K}\left(\mathbf{r}_{\|}^{\prime}, \mathbf{r}_{\|} ; t\right)^{*} .
$$

The gauge given above is special in that $\bar{K}$ is real. This can be seen by noting that a phase increment $(2 e / \hbar) \mathbf{A} \cdot d l=(2 e \boldsymbol{B} / \hbar) z d x$ changes sign if the path is reflected in the plane of symmetry $z=0$. As a consequence

$K\left(\left(\mathbf{r}_{\|}, z\right),\left(\mathbf{r}_{\|}^{\prime}, z^{\prime}\right) ; t\right)=K\left(\left(\mathbf{r}_{\|},-z\right),\left(\mathbf{r}_{\|}^{\prime},-z^{\prime}\right) ; t\right)^{*}$,

so that upon averaging over $z$ and $z^{\prime}$ the imaginary parts of $K$ cancel. For any $\Delta \mathbf{r}_{\|}, \Delta t$ we now define $k\left(\Delta \mathbf{r}_{\|}, \Delta t\right) \equiv \bar{K}\left(\mathbf{r}_{\|}, \mathbf{r}_{\|}+\Delta \mathbf{r}_{\|} ; \Delta t\right)$, which is independent of $\mathbf{r}_{\| \mid}$because of translational invariance. Since $k$ is real, we have from Eq. (3.3)

$$
k\left(\Delta \mathbf{r}_{\|}, \Delta t\right)=k\left(-\Delta \mathbf{r}_{\|}, \Delta t\right) .
$$

One can represent the classical diffusive motion of an electron by a random walk on a lattice with time step $\Delta t$ and random displacements $\Delta \mathbf{r}_{\|}=( \pm \Delta x, \pm \Delta y)$ in the case of the film, or $\Delta \mathbf{r}_{\|}=( \pm \Delta x, 0)$ in the case of the channel. The time step is chosen such that $\tau_{e}, W^{2} / D \ll \Delta t$ $<<\tau_{B}, \tau_{\phi}$. The corresponding step sizes $\Delta x$ and $\Delta y$ equal $\sqrt{2 D \Delta t}$. On the phase relaxation-time scale the random walk will then be an accurate representation of the diffusive motion with diffusion coefficient $D$. It follows from $\mathrm{Eq}$. (3.4), combined with the reflection symmetry in the plane $y=0$, that

$$
\begin{aligned}
k((\Delta x, \Delta y), \Delta t) & =k((-\Delta x,-\Delta y), \Delta t) \\
& =k((-\Delta x, \Delta y), \Delta t)=k((\Delta x,-\Delta y), \Delta t) .
\end{aligned}
$$

Thus $k$ has the same value (say $k_{0}$ ) for each of the random displacements, irrespective of the direction. This is the crux of the argument. It implies that $\bar{K}\left(\mathbf{r}_{\|}, \mathbf{r}_{\|}^{\prime} ; t\right)=k_{0}^{t / \Delta t}$ is independent of initial and final coordinates, as we set out to prove.

The preceding argument also predicts exponential decay of the average phase factor,

$$
\left\langle e^{i \phi(t)}\right\rangle=k_{0}^{t / \Delta t} \equiv e^{-t / \tau_{B}},
$$

where we have defined the phase relaxation time $\tau_{B}$ in terms of $k_{0}$. We now proceed to the calculation of $\tau_{B}$.

\section{ANALYTICAL RESULTS}

The magnetic field dependence of the phase relaxation time $\tau_{B}$ depends on the relative magnitudes of three lengths; the magnetic length $l_{m} \equiv(\hbar / e B)^{1 / 2}$, the film thickness or channel width $W$, and the (bulk) elastic mean-free path $l_{e} \equiv v_{F} \tau_{e}$ (where $v_{F}$ is the Fermi velocity). Analytical expressions for $\tau_{B}$ can be obtained in three asymptotic regimes; dirty metal $\left(l_{e} \ll W \ll l_{m}\right)$; pure metal in a weak field $\left(l_{e} \gg W, l_{m} \gg \sqrt{W l_{e}}\right)$; and pure metal in a strong field $\left(l_{e} \gg W, W \ll l_{m} \ll \sqrt{W l_{e}}\right)$. These regimes are considered separately below. (For a qualitative picture of the various regimes we refer to Refs. 3-5.) Subsequently, we will discuss the effects of nonuniform width and give expressions for the diffusion coefficient.

\section{A. Dirty metal}

This is the case considered by Al'tshuler and Aronov, ${ }^{2}$ who find for a film

$$
\tau_{B}=3 l_{m}^{4} / D W^{2} .
$$

We give an elementary derivation, due to De Gennes and Tinkham, ${ }^{4}$ which shows that Eq. (4.1) holds for the channel geometry as well. If $l_{e} \ll W$, the electron motion from one boundary to the other is diffusive. Between two impurity collisions the phase increment is much smaller than one, and the total phase shift $\phi(t)$ will have a Gaussian distribution over the paths. This implies that

$$
\left\langle e^{i \phi(t)}\right\rangle=\exp \left[-\frac{1}{2}\left\langle\phi^{2}(t)\right\rangle\right]
$$

with

$$
\left\langle\phi^{2}(t)\right\rangle=\left(\frac{2 e}{\hbar}\right)^{2} \int_{0}^{t} d t^{\prime} \int_{0}^{t} d t^{\prime \prime} \sum_{\alpha, \beta}\left\langle A_{\alpha}\left(\mathbf{R}\left(t^{\prime}\right)\right) A_{\beta}\left(\mathbf{R}\left(t^{\prime \prime}\right)\right) v_{\alpha}\left(t^{\prime}\right) v_{\beta}\left(t^{\prime \prime}\right)\right\rangle
$$


Here $\alpha$ and $\beta$ are $x, y, z$ for the film and $x, z$ for the channel. On the time scale $\tau_{e}$ of fluctuations in the direction of the velocity $\mathbf{v}$, variations in $\mathbf{A}$ are negligible, so that we may approximate

$$
\begin{aligned}
\left\langle A_{\alpha}\left(t^{\prime}\right) A_{\beta}\left(t^{\prime \prime}\right)\right. & \left.v_{\alpha}\left(t^{\prime}\right) v_{\beta}\left(t^{\prime \prime}\right)\right\rangle \\
& =\left\langle A_{\alpha}\left(t^{\prime}\right) A_{\beta}\left(t^{\prime \prime}\right)\right\rangle 2 D \delta_{\alpha \beta} \delta\left(t^{\prime}-t^{\prime \prime}\right),
\end{aligned}
$$

which gives

$$
\left\langle\phi^{2}(t)\right\rangle=(2 e / \hbar)^{2} 2 D t\left\langle A^{2}\right\rangle .
$$

The average of $A^{2}=(B z)^{2}$ is simply an average over the sample volume, equal to $\frac{1}{12}(B W)^{2}$, and the result (4.1) is recovered. We recall that the gauge $\mathbf{A}=(B z, 0,0)$ is imposed by the requirement that the average of $e^{i \phi}$ does not depend on the initial and final coordinates of the path; ${ }^{8}$ see Sec. III. The diffusion coefficient $D$ in the dirty metal is given by $\frac{1}{3} v_{F} l_{e}$ for the film (three-dimensional diffusion) and $\frac{1}{2} v_{F} l_{e}$ for the channel (two-dimensional diffusion). We also note that the result (4.1) only holds for magnetic fields satisfying $l_{m} \gg W$, so that the requirement $\tau_{B} \gg W^{2} / D$ is fulfilled. (At stronger fields the effective dimensionality of the sample changes; see Ref. 2.)

\section{B. Pure metal in weak field}

In the regime $l_{e} \gg W, l_{m} \gg \sqrt{W l_{e}}$ the maximum phase increment between two impurity collisions (of order $\left.W l_{e} B e / \hbar\right)$ is much smaller than 1, so that the Gaussian approximation (4.2) still holds. The electrons now move ballistically from one boundary to the other, and consequently the boundary scattering has to be treated explicitly. For a film with diffuse surface scattering Dugaev and Khmel'nitskii ${ }^{3}$ find

$$
\tau_{B}=C_{1} l_{m}^{4} / W^{3} v_{F}
$$

with $C_{1}=16$, in agreement with the calculation of De Gennes and Tinkham ${ }^{4}$ for the superconductivity problem. Using the "method of trajectories" of these latter authors we have calculated $\tau_{B}$ also for the case of specular scattering, and for both film and channel geometries. These calculations are given in the Appendix. The result is still of the form (4.6), but with different values for the coefficient $C_{1}$; see Table $I$. Note that in this weak-field regime one always has $\tau_{B} \gg \tau_{e}$, so that the condition of Sec. II that many impurity collisions take place before phase relaxation does not lead to an additional restriction on the magnetic field strength. ${ }^{9}$

TABLE I. Coefficients $C_{1}$ and $C_{2}$ appearıng in Eqs. (4.6) and (4.7) for the phase relaxation time in the weak- and strong-field regimes, for diffuse and specular boundary scattering in film and channel geometries. The two entries for a film with diffuse scattering were prevıously obtained in Refs. 3 and 4.

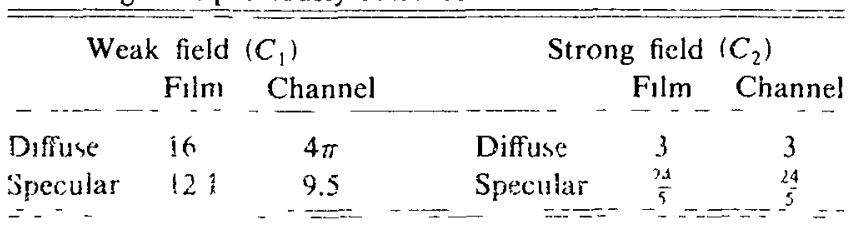

\section{Pure metal in strong field}

In the regime $l_{e} \gg W, l_{m} \ll \sqrt{W l_{e}}$ a phase change of order unity can occur between two impurity collisions, and the Gaussian approximation can no longer be used. For a film with diffuse surface scattering the result is 3,4

$$
\tau_{B}=C_{2} \tau_{e} l_{m}^{2} / W^{2}
$$

with $C_{2}=3$. In the Appendix we calculate ${ }^{10}$ that for specular scattering $C_{2}=\frac{24}{5}$. The coefficient $C_{2}$ is the same in the film and channel geometries. In the present regime the strength of the magnetic field is limited by the requirement $\tau_{B} \gg \tau_{e}$, which implies the restriction $l_{m} \gg W$. Notice the curious dependence of $\tau_{B}$ on the type of scattering (Table I): In weak fields the phase relaxation is faster for specular than for diffuse scattering, whereas in strong fields the situation is reversed.

\section{Nonuniform $\boldsymbol{W}$}

In the calculation of $\tau_{B}$ for a pure metal it is assumed that the boundaries at $z= \pm W / 2$ are perfectly flat. The influence of small spatial variations $\delta$ in $W$ may be estimated as follows. An electron moving ballistically from one boundary to the other acquires a phase increment

$\Delta \phi=\frac{2 e B}{\hbar} \int_{z=-W / 2-\delta_{l}}^{z=+W / 2+\delta_{f}} z d x=W l_{m}^{-2} \frac{v_{x}}{v_{z}}\left(\delta_{f}-\delta_{l}\right)+O\left(\delta^{2}\right)$.

We denote by $\delta_{i}$ and $\delta_{f}$ the variations in the $z$ coordinate of the boundary at the initial and final points. For a typical trajectory with $v_{x}, v_{z} \sim v_{F}$ there are about $t v_{F} / W$ small phase increments $\Delta \phi$ in a time $t$, which lead to an additional contribution to the phase relaxation rate $1 / \tau_{B}$ of $\left\langle(\Delta \phi)^{2}\right\rangle v_{F} / W \sim W \delta^{2} v_{F} l_{m}^{-4}$ [in the Gaussian approximation (4.2)]. In the weak-field regime this correction is relatively small, of $\operatorname{order}(\delta / W)^{2}$. In the strong-field regime the relative correction is larger, of order $(\delta / W)^{2} W l_{e} l_{m}^{-2}$. Thus, for fields such that $l_{m}>W$, random variations in $W$ of rms $\delta$ may be neglected provided $\delta / W \ll\left(W / l_{e}\right)^{1 / 2}$.

\section{E. Diffusion coefficient}

When applying the above results ${ }^{5}$ one also needs expressions for the diffusion coefficient as a function of $W$. In the case of diffuse boundary scattering, one has for a thin film the Fuchs formula"

$D(\mathrm{fim})=\frac{1}{3} v_{F} l_{e}\left(1-\frac{3 l_{e}}{2 W} \int_{0}^{1} d s s\left(1-s^{2}\right)\left(1-e^{-W / s l_{e}}\right)\right)$,

and for a narrow two-dimensional electron gas we find by an analogous calculation

$$
\begin{array}{r}
\left.D(\text { channel })=\frac{1}{2} v_{l} l_{e} \mid \begin{array}{r}
1-\frac{4 l_{c}}{\pi W} \int_{0}^{1} d s s\left(1-s^{2}\right)^{1 / 2} \\
\times\left(1-e^{-W / s l_{e}}\right)
\end{array}\right)
\end{array}
$$


In the limit $l_{e} / W \rightarrow \infty$ these two expressions simplify to $D=$ const $\times v_{F} W \ln \left(l_{e} / W\right)$, where the constant equals $\frac{1}{4}$ for the film and $1 / \pi$ for the channel. In the case of specular scattering, one obviously has $D$ (film) $=\frac{1}{3} v_{F} l_{e}$ and $D$ (channel) $=\frac{1}{2} v_{F} l_{e}$, irrespective of $W$.

\section{NUMERICAL RESULTS}

If the characteristic length scales are comparable, the analytic formulas for $\tau_{B}$ of Sec. IV are inapplicable. To study these intermediate regimes as well, we have numerically simulated the phase relaxation of an electron in a magnetic field. The method used is straightforwardexcept for one point concerning the gauge trick.

An electron starts moving on a straight line with a velocity $v_{F}$ in a random direction, ${ }^{12}$ until it suffers an impurity collision after a time interval $\Delta t$, chosen with probability density $\tau_{e}^{-1} \exp \left(-\Delta t / \tau_{e}\right)$. This process then repeats itself. Whenever the electron hits the boundary at $z= \pm W / 2$ it is reflected either specularly or diffusely. ( In the latter case the direction of reflection is chosen randomly with weight $\cos \theta$, where $\theta$ is the angle with the normal to the boundary, so that the outgoing flux of electrons is isotropic.) Along the path the integral $\int \mathbf{A} \cdot d l$ is calculated, which is simple since the trajectory consists of straight line segments. The resulting phase factor $e^{i \phi(t)}$ [Eq. (2.2)] is averaged over $10^{4}$ electrons. In principle, only those electrons which at time $t$ are at (or near) their starting point at $t=0$ should be considered [Eq. (2.3)]. These are so few that it would be very time consuming to achieve good statistics. Fortunately, the gauge trick says that if we choose $\mathbf{A}=(\boldsymbol{B z}, 0,0)$ we may average over all electrons; see Sec. III. This increases enormously the efficiency of the algorithm.

For $t \gg \tau_{e}, W^{2} / D$ an exponential decay $\left\langle e^{i \phi(t)}\right\rangle \propto e^{-i / \tau_{B}^{e}}$ is found. In the asymptotic regimes the data agree with the analytical formulas within the numerical accuracy. Results for the intermediate regimes are illustrated in Figs. 2 and 3, for the film geometry with specular surface scattering. Figure 2 shows how the phase relaxation time $\tau_{B}$ changes for a fixed magnetic field as we go from a dirty metal $\left(l_{e}<W\right)$ to a pure metal $\left(l_{e} \gg W\right)$. Figure 3 shows for a pure metal the crossover from the weak-field regime $\left(l_{m} \gg \sqrt{l_{e} W}\right)$ to the strong-field regime $\left(W \ll l_{m} \ll \sqrt{l_{e} W}\right)$. We make the following comments on these two figures.

A magnetic field significantly reduces the weak localization of electrons for $B \gtrsim B^{*}$, where the characteristic field strength $B^{*}$ is such that $\tau_{B} \sim \tau_{\phi}$. (This is the field for which the trajectory of an electron returning after a time $\tau_{\phi}$ encloses a flux of order $\hbar / 2 e$.) Figure 2 tells us that $B^{*}$ as a function of $l_{e}$ has a minimum. This feature is fully analogous to the minimum in the parallel critical field of thin superconducting films predicted by De Gennes and Tinkham. ${ }^{4}$ The physics involved is simple: In dirty metals the flux enclosed by a trajectory of duration $\tau_{\phi}$ is proportional to its extension $\sqrt{D \tau_{\phi}} \propto \sqrt{l_{e}}$. As $l_{e}$ goes down $B^{*}$ goes up. In pure metals, on the contrary, the flux enclosed is proportional not to the extension but to the number $\tau_{\phi} / \tau_{e} \propto 1 / l_{e}$ of impurity collisions involved

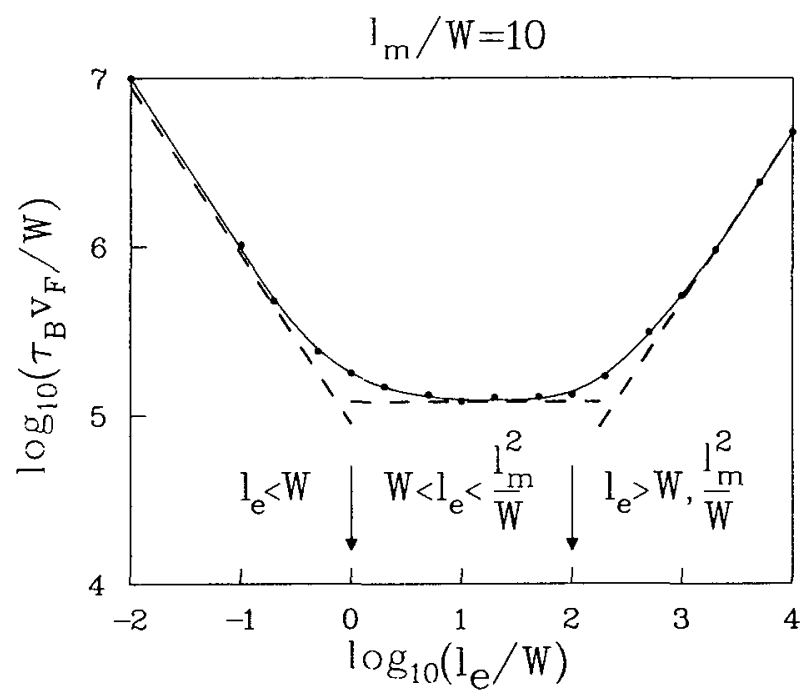

FIG. 2. Phase relaxation time $\tau_{B}$ in a film with specular surface scattering, as a function of the (bulk) elastic mean free path $l_{e}$. This plot is obtained by numerical simulation of the phase relaxation in a parallel magnetic field with $l_{m}=10 \mathrm{~W}$. The dashed lines are analytic formulas valid in the three asymptotic regimes. Arrows indicate the crossover points from one regime to another.

(see Sec. I), so that as $l_{e}$ goes up $B^{*}$ does too.

The crossover from the weak- to the strong-field regime shown in Fig. 3 (for a pure metal) is well described by the interpolation formula

$$
\tau_{B}=\tau_{B}(\text { weak })+\tau_{B}(\text { strong }) .
$$

Here $\tau_{B}$ (weak) and $\tau_{B}$ (strong) are the phase relaxation

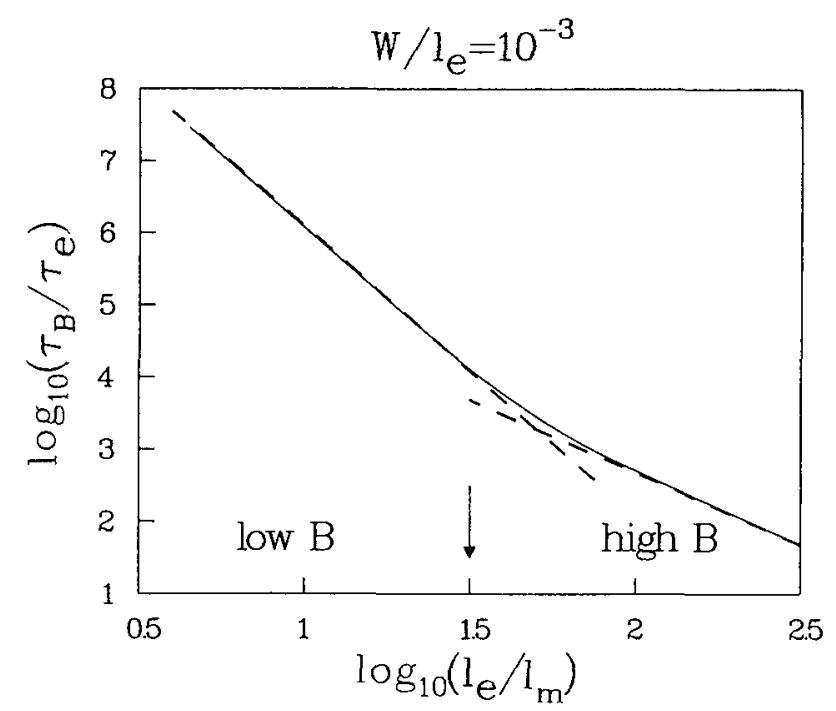

FIG. 3. Phase relaxation time $\tau_{B}$ vs magnetic length $l_{m}$ in a thin film with specular surface scattering (fixed $l_{e}$ and $W=10^{-3} l_{\mathfrak{c}}$ ). The solid curve is obtained by numerical simulation and shows the crossover from the weak-field regime to the strong-field regime in a pure metal (dashed lines are the analytic asymptotic formulas). 
times given by, respectively, Eq. (4.6) and Eq. (4.7). This simple formula is useful in the interpretation of magnetoresistance data. ${ }^{5}$

\section{DISCUSSION}

Recently, the authors in collaboration with Van Wees and Mooij have measured the magnetoresistance of a narrow $\mathrm{GaAs}-\mathrm{Al}_{x} \mathrm{Ga}_{1-x}$ As heterostructure in the highmobility regime $l_{e}>W$. As reported in Ref. 5, it is possible to explain the data for magnetic fields such that $l_{m}>W$ by means of the formulas for a channel with specular boundary scattering obtained in Secs. IV and V. We now discuss our results in relation to experiment.

The weak localization correction to the conductivity depends on the type of boundary scattering via two quantities, the phase relaxation time $\tau_{B}$ and the diffusion coefficient $D$. The order of magnitude of these dependencies is entirely different. Whereas $\tau_{B}$ varies less than a factor of 2 between specular and diffuse scattering, variations in $D$ are much larger ( $D$ is reduced by a factor $W / l_{e}$ ). In the analysis of the experimental data ${ }^{5}$ the rather subtle scattering type dependence of $\tau_{B}$ is lost, and only the more elementary variations in $D$ are seen.

In addition to the weak localization correction (which is a single-particle quantum interference effect) there is a quantum correction to the conductivity arising from electron-electron interactions. ${ }^{1}$ In nonsuperconducting materials this latter effect is insensitive to magnetic fields $B<k_{B} T / g \mu_{B}$ for which the spin splitting may be neglected. This is usually the case in the field range of interest for the weak localization effect. ${ }^{13,14}$ These are all quantum-mechanical effects of a magnetic field on the conductivity. Classical $B$ dependencies may be neglected if the cyclotron radius $m v_{F} / e B$ is much larger than $W$. Classical and quantum effects can be distinguished experimentally by raising the temperature, thereby suppressing the quantum contributions to the magnetoresistance. ${ }^{5,14,15}$

Very recently several other groups have reported magnetoresistance experiments on narrow high-mobility GaAs- $\mathrm{Al}_{x} \mathrm{Ga}_{1-x}$ As heterostructures. ${ }^{16-18}$ The theoretical expressions presented in this paper may also be relevant to part of their data. We would like to emphasize, however, that the applicability of these expressions is restricted to channels long enough for the diffusive approximation to hold. The multiterminal samples currently studied allow the voltage drop to be measured over very short channel sections, shorter than the phase coherence length, or even the elastic mean-free path. ${ }^{17,19}$ Under such circumstances the concept of a conductivity loses its meaning. It would be of interest to study the quantum interference effects in this ballistic regime theoretically.

We mention several other directions in which one might extend the analysis. The first is to magnetic fields such that $l_{m}<W$. Then $\tau_{B}$ becomes less than $\tau_{e}$ and the approximation of diffusive motion used here breaks down. Another difficulty encountered in such strong fields is that even small variations in width along the channel will lead to a significant phase relaxation, since the exact cancellation of phase increments (Fig. 1) no longer holds. The order of magnitude of this effect is estimated in Sec. IV. ${ }^{20}$ A second direction in which to extend the analysis is to include quantum size effects on the magnetoresistance. ${ }^{21}$ These effects certainly play a role in semiconductor channels with only a few one-dimensional subbands occupied, ${ }^{5,16-18}$ but go beyond the present semiclassical theory. Finally, we mention that boundary scattering affects the quantumfluctuations ${ }^{22,23}$ in the magnetoresistance in much the same way as it affects weak localization; see Ref. 24.

\section{APPENDIX: CALCULATION OF $\tau_{B}$ BY DE GENNES AND TINKHAM'S METHOD OF TRAJECTORIES}

\section{A. Pure metal in weak field}

We first consider a film with specular surface scattering. We compute separately the phase increments $\Delta \phi$ acquired by an electron as it moves along a straight line from an impurity to a wall $(\Delta \phi)_{i w}$, from a wall to an impurity $(\Delta \phi)_{w i}$, from one impurity to another $(\Delta \phi)_{i i}$, and from one wall to the opposite wall $(\Delta \phi)_{w w}$. The phase shift along a straight line segment is given by $2 l_{m}^{-2} \int_{z_{1}}^{z_{2}} z d x=l_{m}^{-2}\left(z_{2}^{2}-z_{1}^{2}\right) \cot \alpha$. Here $z_{1}$ and $z_{2}$ are the initial and final $z$ coordinates of the segment and $\alpha$ is the angle with the $x$ axis of its projection on the $x-z$ plane. One thus finds

$$
\begin{aligned}
& (\Delta \phi)_{i w}=l_{m}^{-2}\left(\frac{1}{4} W^{2}-z_{1}^{2}\right) \cot \alpha, \\
& (\Delta \phi)_{w i}=l_{m}^{-2}\left(z_{2}^{2}-\frac{1}{4} W^{2}\right) \cot \alpha, \\
& (\Delta \phi)_{i i}=l_{m}^{-2}\left(z_{2}^{2}-z_{1}^{2}\right) \cot \alpha, \\
& (\Delta \phi)_{w w}=0 .
\end{aligned}
$$

The last equation expresses the flux cancellation on trajectories without impurity scattering; see Fig. 1.

In the limit $W / l_{e} \rightarrow 0$ one can easily calculate the average square of these phase increments (see Ref. 4) and the result is

$$
\left\langle(\Delta \phi)_{i w}^{2}\right\rangle=\left\langle(\Delta \phi)_{w i}^{2}\right\rangle=\left\langle(\Delta \phi)_{i i}^{2}\right\rangle=\frac{1}{24} l_{m}^{-4} l_{c} W^{3} .
$$

To calculate the average square of the total phase shift along a trajectory [the quantity which determines $\tau_{B}$ in the weak-field regime according to Eq. (4.2)], one has to also know the average product of the phase increments along two different segments. This average vanishes if the angles $\alpha_{1}$ and $\alpha_{2}$ of the two segments are uncorrelated. Since an impurity collision destroys the angular correlations, the only cross term one has to consider is $\Gamma \equiv\left\langle(\Delta \phi)_{i w}(\Delta \phi)_{w i}\right\rangle$, corresponding to a trajectory starting and ending at an impurity with one or more wall collisions in between. If $p$ is the number of wall collisions then the angles $\alpha_{1}$ and $\alpha_{2}$ are related by $\alpha_{2}=(-1)^{p} \alpha_{1}$, for the case of specular scattering considered. The statistical weight of the trajectory is 
$\left(4 \pi W l_{e}\right)^{-1} \sin \theta d \theta d \alpha_{1} d z_{1} d r_{2}$

$$
\times \exp \left\{-l_{e}^{-1}\left[r_{1}+r_{2}+(p-1) r_{0}\right]\right\} .
$$

(Here $\theta$ is the angle with the $y$ axis, $z_{1}$ and $z_{2}$ are the ini- tial and final $z$ coordinates, the initial and final straight line segments have lengths $r_{1}$ and $r_{2}$, and each of the $p-1$ segments linking one wall to the other has length $r_{0}$; see Fig. 4.) The expression for $\Gamma$ becomes

$$
\begin{aligned}
\Gamma=\left(\pi W l_{e} l_{m}^{4}\right)^{-1} \int_{0}^{\pi} d \theta \sin \theta \sum_{p=1}^{\infty} \int_{-W / 2}^{+W / 2} d z_{1} \int_{0}^{\pi / 2} d \alpha_{1} \int_{0}^{r_{0}} d r_{2}\{-1)^{p} \cot ^{2} \alpha_{1}\left(\frac{1}{4} W^{2}-z_{1}^{2}\right)\left(z_{2}^{2}-\frac{1}{4} W^{2}\right) \\
\\
\times \exp \left\{-l_{e}^{-1}\left[r_{1}+r_{2}+(p-1) r_{0}\right]\right\} .
\end{aligned}
$$

For $l_{e} \gg W$ the dominant contributions to this integral come from grazing trajectories with $\alpha_{1}$ close to zero, so that we may approximate $\cot \alpha_{1}$ by $1 / \alpha_{1}$. Expressing $z_{2}, r_{0}$, and $r_{1}$ in terms of $\theta, z_{1}, \alpha_{1}$, and $r_{2}$ we find (omitting subscripts)

$$
\begin{aligned}
& \Gamma=\left(\pi W l_{e} l_{m}^{4}\right)^{-1} \int_{0}^{\pi} d \theta \sin \theta \sum_{p=1}^{\infty} \int_{-W / 2}^{+W / 2} d z \int_{0}^{\pi / 2} d \alpha \int_{0}^{W / \alpha \sin \theta} d r(-1)^{p} \alpha^{-2}\left(\frac{1}{4} W^{2}-z^{2}\right) r \alpha \sin \theta(r \alpha \sin \theta-W) \\
& \times \exp \left[-r / l_{e}-\left(p W-\frac{1}{2} W-z\right) / l_{e} \alpha \sin \theta\right] .
\end{aligned}
$$

Upon integration over $r$ and $\alpha$ this reduces to

$$
\Gamma=\frac{4}{\pi} \overline{\sin \theta} l_{m}^{-4} l_{e} \sum_{p=1}^{\infty} \int_{-W / 2}^{+W / 2} d z(-1)^{p}\left(\frac{1}{4} W^{2}-z^{2}\right)\left[1+(p-z / W) \ln \left(\frac{p W-W / 2-z}{p W+W / 2-z}\right)+O\left(W / l_{e}\right)\right\},
$$

with the average $\overline{\sin \theta} \equiv \frac{1}{2} \int_{0}^{\pi} \sin ^{2} \theta d \theta=\pi / 4$. Finally, integration over $z$ and a numerical evaluation of the series in $p$ gives the answer

$$
\Gamma=\gamma l_{m}^{-4} l_{e} W^{3}, \text { with } \gamma=0.0200 .
$$

Collecting results, we find that the average square phase shift after $n$ impurity collisions is given by

$$
\begin{aligned}
\left\langle\phi^{2}\right\rangle & =n\left\langle(\Delta \phi)_{i \omega}^{2}+(\Delta \phi)_{w i}^{2}+(\Delta \phi)_{i i}^{2}+2(\Delta \phi)_{\omega}(\Delta \phi)_{w i}\right\rangle \\
& =n\left(\frac{1}{8}+2 \gamma\right) l_{m}^{-4} l_{e} W^{3} .
\end{aligned}
$$

The trajectories contributing to this average have duration $t=n \tau_{e}\left[1+O\left(n^{-1 / 2}\right)\right]$. For $n \gg 1$ we can put $n=t / \tau_{e}$ to obtain

$$
\left\langle e^{i \phi(t)}\right\rangle=\exp \left[-\left(\frac{1}{16}+\gamma\right) v_{F} W^{3} I_{m}^{-4} t\right],
$$

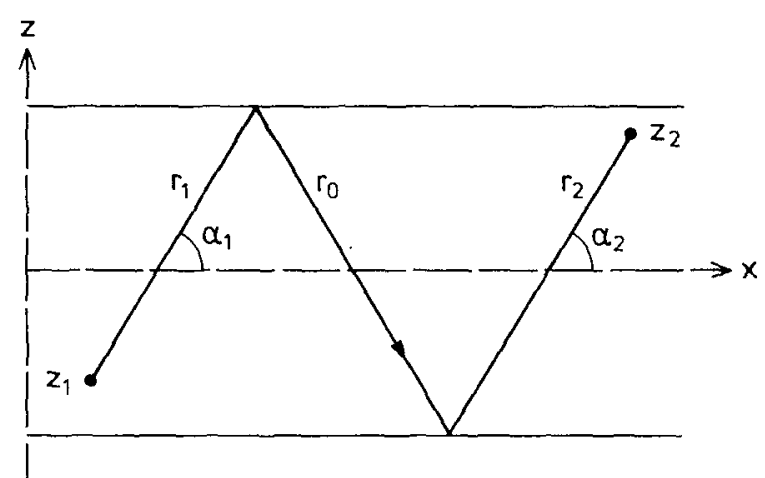

FIG. 4. Sketch of trajectory contributing to the correlation term $\Gamma$ in the case of specular boundary scattering, illustrating the symbols defined in the text. The straight line segments make an angle $\theta$ with the $y$ axis; shown is the projection on the $x-z$ plane. by virtue of Eq. (4.2). The phase relaxation time $\tau_{B}$ is then given by Eq. (4.6) with $C_{1}=16(1+16 \gamma)^{-1}=12.1$. This result is derived for a film with specular surface scattering. Diffuse scattering destroys the angular correlation responsible for the term $\gamma$, so that we recover the result $C_{1}=16$ of Refs. 3 and 4 . The results for $\left\langle\phi^{2}\right\rangle$ in the channel geometry follow immediately from the preceding expressions, upon multiplication with $1 / \overline{\sin \theta}=4 / \pi$ [see, for example, Eq. (A5)]. We thus find $C_{1}=4 \pi(1+16 \gamma)^{-1}$ for specular scattering, and $C_{1}=4 \pi$ for diffuse scattering.

\section{B. Pure metal in strong field}

In this regime we cannot use the Gaussian formula (4.2), but have to calculate the average phase factor itself. The calculation is simplified by the fact that $l_{e}$ may be taken infinitely large (since $l_{e}$ is much larger than the other characteristic lengths $W$ and $l_{m}^{2} / W$ ). We first consider the case of specular boundary scattering in either the film or channel geometry.

Let $z_{k}(k=1,2, \ldots, n)$ be the $z$ coordinate of the $k$ th impurity collision on a trajectory, and $\beta_{k}$ and $\alpha_{k}$, respectively, the angles before and after scattering (these are the angles which the projection of the trajectory on the $x-z$ plane makes with the $x$ axis). The phase shift consists of alternating $i w$ and $w i$ increments (the $i i$ increments have become negligibly few),

$\phi=l_{m}^{-2} \sum_{k=1}^{n}\left[\left(\frac{1}{4} W^{2}-z_{k}^{2}\right) \cot \alpha_{k}+\left(z_{k+1}^{2}-\frac{1}{4} W^{2}\right) \cot \beta_{k+1}\right]$.

For specular scattering $\beta_{k+1}$ equals either plus or minus $\alpha_{k}$, each with probability $\frac{1}{2}$. The average phase factor is given by 


$$
\begin{array}{r}
\left\langle e^{i \phi}\right\rangle=\left\langle\prod _ { k = 1 } ^ { n } \frac { 1 } { 2 } \left\{\exp \left[i l_{m}^{-2}\left(z_{k+1}^{2}-z_{k}^{2}\right) \cot \alpha_{k}\right]\right.\right. \\
+\exp \left[i l _ { m } ^ { - 2 } \left(\frac{1}{2} W^{2}-z_{k}^{2}\right.\right. \\
\left.\left.\left.\left.-z_{k+1}^{2}\right) \cot \alpha_{k}\right]\right\}\right),
\end{array}
$$

which, upon averaging over $\alpha$, takes the form

$$
\begin{aligned}
\left\langle e^{\prime \phi}\right\rangle=\left\langle\prod_{k=1}^{n} \frac{1}{2}\{\right. & \exp \left(-l_{m}^{-2}\left|z_{k+1}^{2}-z_{k}^{2}\right|\right) \\
& \left.\left.\quad+\exp \left[-l_{m}^{-2}\left(\frac{1}{2} W^{2}-z_{k}^{2}-z_{k+1}^{2}\right)\right]\right\}\right) .
\end{aligned}
$$

For $l_{m} \gg W$ we may expand the exponentials,

$$
\begin{aligned}
\left\langle e^{l \phi}\right\rangle= & {\left[1-\frac{1}{4} W^{2} l_{m}^{-2}+O\left(W / l_{m}\right)^{4}\right]^{n} } \\
& \times\left\langle\prod_{k=1}^{n}\left(1+\epsilon_{k, k+1}\right)\right\rangle,
\end{aligned}
$$

where $\epsilon_{k, k+1} \equiv l_{m}^{-2} \min \left(z_{k}^{2}, z_{k+1}^{2}\right)$ is of order $\left(W / l_{m}\right)^{2}$. We need to compute the average over $z$ in the limit $\epsilon \rightarrow 0$, $n \rightarrow \infty$, with $n \in$ remaining finite. This can be done as follows. The average of $\epsilon$ equals

$$
\begin{aligned}
\left\langle\epsilon_{12}\right\rangle & \equiv \int_{-W / 2}^{+W / 2} \frac{d z_{1}}{W} \int_{-W / 2}^{+W / 2} \frac{d z_{2}}{W} l_{m}^{-2} \min \left(z_{1}^{2}, z_{2}^{2}\right) \\
& =\frac{1}{24} W^{2} l_{m}^{-2} .
\end{aligned}
$$

We may therefore write

$$
\left\langle\prod_{k=1}^{n}\left(1+\epsilon_{k, k+1}\right)\right\rangle=\left(1+\frac{1}{24} W^{2} l_{m}^{-2}\right)^{n}\left\langle\prod_{k=1}^{n}\left(1+\epsilon_{k, k+1}^{\prime}\right)\right\rangle,
$$

where $\left\langle\epsilon_{k, k+1}^{\prime}\right\rangle=0$. Expanding the product over $k$ in powers of $\epsilon^{\prime}$ we have, because only correlated products of $\epsilon^{\prime}$ contribute upon averaging,

$$
\begin{aligned}
\left\langle\prod_{k=1}^{n}\left(1+\epsilon_{k, k+1}^{\prime}\right\rangle=\right. & 1+(n-1)\left\langle\epsilon_{12}^{\prime} \epsilon_{23}^{\prime}\right\rangle \\
& +(n-2)\left\langle\epsilon_{12}^{\prime} \epsilon_{23}^{\prime} \epsilon_{34}^{\prime}\right\rangle+\cdots .
\end{aligned}
$$

In the limit $\epsilon \rightarrow 0, n \epsilon$ finite, only the leading 1 in the expansion survives, so that

$$
\left\langle e^{l \phi}\right\rangle=\left(1-\frac{5}{24} W^{2} l_{m}^{-2}\right)^{n}=\exp \left(-\frac{5}{24} n W^{2} l_{m}^{-2}\right)
$$

in this limit. Putting $n=t / \tau_{e}$ as above, it follows that $\tau_{B}$ is given by Eq. (4.7) with $C_{2}=\frac{24}{5}$. Note that this result holds for both film and channel geometries, since the angle $\theta$ does not appear in the preceding equations. (This is a consequence of the fact that, if impurity collisions are rare, only the projection of the trajectories on the $x-z$ plane matters for the phase relaxation rate, and not their actual length.)

In the case of diffuse boundary scattering we do not have the correlation between the angles $\alpha$ and $\beta$ which complicated the preceding calculation for specular scattering. By rearranging terms in Eq. (A9) the average phase factor can be written as

$$
\left\langle e^{i \phi}\right\rangle=\left\langle\prod_{k=1}^{n} \exp \left[i l_{m}^{-2}\left(z_{k}^{2}-\frac{1}{4} W^{2}\right)\left(\cot \beta_{k}-\cot \alpha_{k}\right)\right]\right\rangle \text {. }
$$

The averages over $\alpha$ and $\beta$ may be carried out independently, which gives

$\left\langle e^{\mid \phi}\right\rangle=\left[\int_{-W / 2}^{+W / 2} \frac{d z}{W} \exp \left[2 l_{m}^{-2}\left(z^{2}-\frac{1}{4} W^{2}\right)\right]\right]^{n}$.

In the limit $W / l_{m} \rightarrow 0, n\left(W / l_{m}\right)^{2}$ finite, this reduces to

$$
\left\langle e^{\prime \phi}\right\rangle=\left(1-\frac{1}{3} W^{2} l_{m}^{-2}\right)^{n}=\exp \left(-\frac{1}{3} n W^{2} l_{m}^{-2}\right) .
$$

With $n=t / \tau_{e}$ we thus recover the result $\tau_{B}=3 \tau_{e} l_{m}^{2} W^{-2}$ of Refs. 3 and 4.
${ }^{1}$ See the review articles by P. A. Lee and T. V. Ramakrishnan, Rev. Mod. Phys. 57, 287 (1985); B. L. Al'tshuler and A. G. Aronov, in Electron-Electron Interactions in Disordered Systems, edited by A. L. Efros and M. Pollak (North-Holland, Amsterdam, 1985), p. 1; H. Fukuyama, ibid., p. 155.

${ }^{2}$ B. L. Al'tshuler and A. G. Aronov, Pis'ma Zh. Eksp. Teor. Fiz. 33, 515 (1981) [JETP Lett. 33, 499 (1981)].

${ }^{3}$ V. K. Dugaev and D. E. Khmel'nitskii, Zh. Eksp. Teor. Fiz. 86, 1784 (1984) [Sov. Phys._JETP 59, 1038 (1984)].

${ }^{4}$ P. G. de Gennes and M. Tinkham, Physics (N.Y.) 1, 107 (1964); see also P. G. de Gennes, Superconductivity of Metals and Alloys (Benjamin, New York, 1966), Ch. 8.

${ }^{5} \mathrm{H}$. van Houten, C. W. J. Beenakker, B. J. van Wees, and J. E. Mooij, Surf. Sci. 196, 144 (1988); H. van Houten, C. W. J. Beenakker, M. E. I. Broekaart, M. G. J. Heijman, B. J. van Wees, J. E. Mooij, and J. P. André, Acta Electron. (to be published).

${ }^{6} \mathrm{~S}$. Chakravarty and A. Schmid, Phys. Rep. 140, 193 (1986).

${ }^{7} \mathrm{~A}$ step function $\theta\left(t-\tau_{e}\right)$ is an alternative choice for the short- time cutoff (used, for example, in Ref. 6). The smooth cutoff $1-\exp \left(-t / \tau_{e}\right)$ used in obtaining Eq. (2.4) provides a better description of the data if $\tau_{\phi}$ is comparable to $\tau_{e}$ (as in Ref. 5). In the limit $\tau_{\phi} / \tau_{e} \rightarrow \infty$ both cutoffs are equivalent.

${ }^{8}$ In the problem of de Gennes and Tinkham (Ref. 4) this same gauge follows upon requiring that the superconductivity order parameter is constant in space.

${ }^{9}$ This condition also requires $\tau_{\phi} \gg \tau_{e}$ or, equivalently, $v_{F} \tau_{\phi} \gg l_{e}$. DK (Ref. 3) instead write $\sqrt{D \tau_{\phi}} \gg l_{e}$. Their much stronger requirement is not borne out by our analysis. A similar disagreement with DK exists in the strong-field regime (Sec. IV C).

${ }^{10}$ This exact value of $C_{2}$ is slightly more accurate than the numerical value reported by us in Ref. 5 .

${ }^{11}$ K. Fuchs, Proc. Cambridge Philos. Soc. 34, 100 (1938); E. H. Sondheimer, Adv. Phys. 1, 1 (1952).

${ }^{12} \mathrm{An}$ efficient algorithm to generate random directions is given by G. Marsaglia, Ann. Math. Stat. 43, 645 (1972).

${ }^{13}$ In this field range the magnetoresistance is an orbital effect 
and quantum corrections from electron-electron interactions contribute only via the so-called Cooper channel.' Relative to the weak localization contribution this is of order

$$
\lambda\left[1+\lambda \ln \left(T_{F} / T\right)\right]^{-1}\left(\hbar / k_{B} T \tau_{\phi}\right)^{1 / 2},
$$

where $T_{F}$ is the Fermi temperature and the coupling constant $\lambda$ is of order unity for $\mathrm{GaAs}$. In the GaAs- $\mathrm{Al}_{x} \mathrm{Ga}_{1-x}$ As heterostructure studied in Ref. $5\left(T_{F}=105 \mathrm{~K}, T=4 \mathrm{~K}\right.$, $\left.\hbar / k_{B} \tau_{\phi}=1.5 \mathrm{~K}\right)$ this would be a $10 \%$ correction, which is ignored.

${ }^{14}$ K. K. Choi, D. C. Tsui, and S. C. Palmateer, Phys. Rev. B 33, 8216 (1986).

${ }^{15}$ Choi et al. (Ref. 14) discovered a temperature-independent negative magnetoresistance, occurring when the cyclotron radius becomes comparable to $W$. We have attributed this classical effect to reduced backscattering in a magnetic field; see H. van Houten, C. W. J. Beenakker, P. H. M. van Loosdrecht, T. J. Thornton, H. Ahmed, M. Pepper, C. T. Foxon, and J. J. Harris, Phys. Rev. B 37, 8534 (1988).

${ }^{16}$ A. M. Chang, G. Timp. T. Y. Chang, J. E. Cunningham, B. Chelluri, P. M. Mankiewich, R. E. Behringer, and R. E. Howard, Surf. Sci. 196, 46 (1988); A. M. Chang, G. Timp. R. E.
Howard, R. Behringer, P. M. Mankiewich, J. E. Cunningham, T. Y. Chang, and B. Chelluri, Superlattices Microstruct. (to be published).

${ }^{17}$ M. L. Roukes, A. Scherer, S. J. Allen Jr., H. G. Craighead, R. M. Ruthen, E. D. Beebe, and J. P. Harbison, Phys. Rev. Lett. 59, 3011 (1987)

${ }^{18}$ J. A. Simmons, D. C. Tsui, and G. Weimann, Surf. Sci. 196, 81 (1988).

${ }^{19}$ G. Timp, A. M. Chang, P. Mankiewich, R. Behringer, J. E. Cunningham, T. Y. Chang, and R. E. Howard, Phys. Rev. Lett. 59, 732 (1987).

${ }^{20}$ Variations in $W$ of order $\delta$ can be neglected if $\delta^{2} l_{e} / W l_{m}^{2} \ll 1$. In Ref. 5 it is estimated that $l_{e}=350 \mathrm{~nm}, W=100 \mathrm{~nm}$, so that in the regime $l_{m}>W$ some 10 or $20 \%$ variations in width should be acceptable.

${ }^{21}$ Z. Tesanovic, M. V. Jaric, and S. Maekawa, Phys. Rev. Lett. 57, 2760 (1986).

${ }^{22}$ B. L. Al'tshuler, Pis'ma Zh. Eksp. Teor. Fiz. 41, 530 (1985) [JETP Lett. 41, 648 (1985)].

${ }^{23}$ P. A. Lee and A. D. Stone, Phys. Rev. Lett. 55, 1622 (1985).

${ }^{24}$ C. W. J. Beenakker and H. van Houten, Phys. Rev. B 37, 6544 (1988). 